\title{
AN APPLICATION OF THE MULTIVARIATE \\ LAGRANGE-BÜRMANN EXPANSION \\ IN MATHEMATICAL GEODESY*
}

\author{
BY \\ P. HENRICI AND G. R. WILKENS \\ University of North Carolina
}

\begin{abstract}
In the simplified model of geodesy where the earth is conceived as a rotational ellipsoid, if the eccentricity of the ellipsoid is to be determined from gravity measurements, an equation of the form $y=x-z h(x)$ is to be solved for $x$, where $y$ and $z$ are small parameters whose values can be measured and $h$ is a known function. We obtain the expansion of $x$ in powers of $y$ and $z$ by means of the general Lagrange-Bürmann formula.
\end{abstract}

1. The problem. Using the standard notations of physical geodesy,

$$
a=\text { major axis of the earth ellipsoid, }
$$

$G M=$ product of the earth's mass and the gravitational constant,

$J_{2}=$ a constant in the expansion of the normal gravity

field in spherical harmonics, and

$\omega=$ angular velocity of the earth,

the equation satisfied by the eccentricity $e$ of the ellipsoid may be stated as follows $[1,4]$ :

$$
3 J_{2}=e^{2}-\frac{4}{15} \frac{\omega^{2} a^{3}}{G M} \frac{e^{3}}{2 q_{0}} .
$$

Here $2 q_{0}$ is a known function of $e$,

$$
2 q_{0}=\left(1+3 / e^{\prime 2}\right) \arctan e^{\prime}-3 / e^{\prime},
$$

where

$$
e^{\prime}=e / \sqrt{1-e^{2}}
$$

\footnotetext{
* Received December 4, 1985.
} 
is the "second eccentricity." The constants $a, G M, J_{2}, \omega$ are either known or can be obtained accurately from gravity measurements. Equation (1) thus serves to obtain accurate values of $e$ from gravity measurements. Our concern is with solving the equation and with exhibiting the dependence of the solution on the parameters.

The equation has the form

$$
y=x-z h(x)
$$

where

$$
y=3 J_{2}, \quad z=\frac{\omega^{2} a^{3}}{G M},
$$

are known and $x=e^{2}$ is to be determined. The function

$$
h(x)=\frac{4}{15} \frac{x^{3 / 2}}{2 q_{0}(\sqrt{x})}
$$

is known. In the physical problem on hand, the numerical values of $y$ and $z$ are both of the order of $3 \times 10^{-3}$.

2. Numerical solution of the equation. This is discussed very thoroughly in [1], and values of $e$ are obtained that are more accurate than those given in the literature. It follows from Eq. (4) of [1] that

$$
\frac{1}{h(x)}=F\left(\frac{3}{2}, \frac{3}{2} ; \frac{7}{2} ; x\right),
$$

where $F$ is the hypergeometric function. Thus $h$ is analytic not only for $0<x<1$ but also at $x=0$. Moreover, since all coefficients in the series (6) are positive, as $x$ increases from 0 to $1, h(x)$ decreases from $h(0)=1$ to $h(1)=4 / 15 \pi$. By writing (4) as a fixed point equation,

$$
x=y+z h(x)
$$

we see that for positive $y$ and $z$ such that $y+z<1$ the equation has precisely one solution, which, if $z$ satisfies the additional condition

$$
\left|z h^{\prime}(y+z)\right|<1
$$

can be found as the limit of the iteration sequence defined by $x_{0}=0$,

$$
x_{n+1}=y+z h\left(x_{n}\right), \quad n=0,1,2, \ldots .
$$

The only numerical problem that arises is a considerable loss of accuracy, due to subtracting large numbers that are nearly equal, if $h$ is evaluated by means of the defining relations (5) and (2). It is much preferable to compute $h$ from the series expansion (6), which converges rapidly if $x$ is small.

3. Analytical solution. Iteration does furnish a numerical solution of (4) for given $y$ and $z$, but it does not show how this solution depends on the parameters. We therefore endeavor to find a series solution for (4). Our tool is the multidimensional Lagrange-Bürmann formula as discussed in [3]. We summarize these results briefly for convenience. 
Let $\mathbf{P}=\left(P_{1}, P_{2}, \ldots, P_{n}\right)$ be an admissible system of $n$ power series in $n$ indeterminates $\mathbf{x}=\left(x_{1}, x_{2}, \ldots, x_{n}\right)$. ["Admissible" means that $P_{j}=c_{j} x_{j}+$ higher-order terms, where $c_{j} \neq 0$.] Let $\mathbf{Q}$ denote the inverse system of $\mathbf{P}$. ["Inverse" means that $\mathbf{Q}$ substituted into $\mathbf{P}$ yields $\mathbf{x}$.] Let $R$ be an arbitrary (single) Laurent series in $\mathbf{x}$. Then the series obtained by substituting $\mathbf{Q}$ into $R$ is given by

$$
R \circ \mathbf{Q}=\sum_{\mathbf{k}} \operatorname{Res}\left(R \mathbf{P}^{-\mathbf{k}-\mathbf{e}} \mathbf{P}^{\prime}\right) \mathbf{x}^{\mathbf{k}},
$$

where the summation is with respect to all index vectors $\mathbf{k}=\left(k_{1}, k_{2}, \ldots, k_{n}\right)$, and where

$$
\begin{gathered}
\mathbf{x}^{\mathbf{k}}=x_{1}^{k_{1}} x_{2}^{k_{2}} \cdots x_{n}^{k_{n}}, \\
\mathbf{e}=(1,1, \ldots, 1),
\end{gathered}
$$

$\mathbf{P}^{\prime}$ is the Jacobian determinant of the system $\mathbf{P}$, and Res denotes the residue, that is, the coefficient of $\mathbf{x}^{-\mathbf{e}}$, in a Laurent series. The result (8) holds formally, that is, regardless of whether or not the series involved are convergent.

We require an application of (8), also given in [3]. Here we consider two systems of complex variables,

$$
\mathbf{x}=\left(x_{1}, \ldots, x_{p}\right), \quad \mathbf{y}=\left(y_{1}, \ldots, y_{q}\right),
$$

and a system of $p$ functions

$$
f_{i}(\mathbf{x}, \mathbf{y}), \quad i=1,2, \ldots, p,
$$

analytic near $(\mathbf{0}, \mathbf{0})$. We write $\mathbf{f}=\left(f_{1}, \ldots, f_{p}\right)$, and we denote by $\mathbf{f}^{\prime}$ the Jacobian determinant of this system with respect to the $x_{i}$, regarding the $y_{j}$ as parameters. Assuming

$$
\mathbf{f}(\mathbf{0}, \mathbf{0})=\mathbf{0}, \quad \mathbf{f}^{\prime}(\mathbf{0}, \mathbf{0}) \neq \mathbf{0},
$$

the system of equations

$$
\mathbf{f}(\mathbf{x}, \mathbf{y})=\mathbf{0}
$$

for sufficiently small $\left|y_{j}\right|$ has precisely one solution $\mathbf{x}(\mathbf{y})$ which is analytic in $\mathbf{y}$ and which satisfies $\mathbf{x}(\mathbf{0})=\mathbf{0}$. We wish to find the coefficients of the power series $\mathbf{x}(\mathbf{y})$ or, more generally, of $r(\mathbf{x}(\mathbf{y}), \mathbf{y})$, where $r$ is a given analytic function.

For a solution by means of the Lagrange-Bürmann formula we assume, without loss of generality, that the matrix

$$
\left(\frac{\partial f_{i}}{\partial x_{j}}(\mathbf{0}, \mathbf{0})\right), \quad i, j=1, \ldots, p,
$$

is the identity. (This can be achieved by forming suitable linear combinations of the functions $f_{i}$ and of the variables $x_{j}$.) In the power series expansion of $\mathbf{f}(\mathbf{x}, \mathbf{y})$, let $\mathbf{B y}$ denote the terms that are linear in the $y_{j}$, that is,

$$
\mathbf{f}(\mathbf{x}, \mathbf{y})=\mathbf{x}+\mathbf{B y}+\text { terms of degree } \geqslant 2 .
$$

(B is a matrix with $p$ rows and $q$ columns; we think of $\mathbf{y}$ as a column vector.) Consider the map of a $(p+q)$-dimensional neighborhood of $(\mathbf{0 , 0})$ defined by

$$
\left(\begin{array}{l}
\mathbf{u} \\
\mathbf{v}
\end{array}\right)=\left(\begin{array}{c}
\mathbf{f}(\mathbf{x}, \mathbf{y})-\mathbf{B y} \\
\mathbf{y}
\end{array}\right)
$$


The system of $p+q$ power series representing this map near $(\mathbf{0 , 0})$ is admissible; in fact, its Jacobian matrix at $(\mathbf{0}, \mathbf{0})$ is the identity. Hence the inverse system

$$
\left(\begin{array}{l}
\mathbf{x} \\
\mathbf{y}
\end{array}\right)=\left(\begin{array}{l}
\mathbf{x}(\mathbf{u}, \mathbf{v}) \\
\mathbf{y}(\mathbf{u}, \mathbf{v})
\end{array}\right)
$$

exists and can be represented by the Lagrange-Bürmann series. Letting

$$
\mathbf{P}=\mathbf{f}(\mathbf{x}, \mathbf{y})-\mathbf{B y},
$$

and noting that the Jacobian determinant of the whole system (10) is just $\mathbf{P}^{\prime}$, the Jacobian with respect to $\mathbf{x}$, one obtains in view of $\mathbf{y}=\mathbf{v}$ for an arbitrary function $r$

$$
r(\mathbf{x}(\mathbf{u}, \mathbf{v}), \mathbf{v})=\sum_{\substack{\mathbf{k} \in Z^{p} \\ \mathbf{m} \in Z^{q}}} \operatorname{Res}\left\{r(\mathbf{x}, \mathbf{y}) \mathbf{P}^{-\mathbf{k}-\mathbf{e}} \mathbf{y}^{-\mathbf{m}-\mathbf{e}} \mathbf{P}^{\prime}(\mathbf{x}, \mathbf{y})\right\} \mathbf{u}^{\mathbf{k}} \mathbf{v}^{\mathbf{m}}
$$

Now evidently $\mathbf{f}(\mathbf{x}, \mathbf{y})=\mathbf{0}$ if and only if $\mathbf{u}=-\mathbf{B v}$. Since $\mathbf{v}=\mathbf{y}$, the solution of (8) thus is

$$
\mathbf{x}(\mathbf{y})=\mathbf{x}(-\mathbf{B y}, \mathbf{y})
$$

and from (12) we find the explicit series expansion

$$
r(\mathbf{x}(\mathbf{y}), \mathbf{y})=\sum_{\substack{\mathbf{k} \in Z^{p} \\ \mathbf{m} \in Z^{q}}} \operatorname{Res}\{\cdots\}(-\mathbf{B y})^{\mathbf{k}} \mathbf{y}^{\mathbf{m}}
$$

where the residues are the same as in (12).

4. Application to the geodesic equation. To apply (13) to the solution of (4), we let $p=1, q=2$,

$$
\mathbf{x}=(x), \quad \mathbf{y}=\left(\begin{array}{l}
y_{1} \\
y_{2}
\end{array}\right)=\left(\begin{array}{l}
y \\
z
\end{array}\right) .
$$

The equation to be solved is $f(\mathbf{x}, \mathbf{y})=0$, where

$$
f(\mathbf{x}, \mathbf{y})=x-y-z h(x),
$$

which in order to isolate first-order terms we write in the form

$$
f(\mathbf{x}, \mathbf{y})=x-y-z-z x g(x)
$$

where

$$
g(x)=\frac{1}{x}(h(x)-1)=O(1)
$$

We see that

$$
\mathbf{B y}=-y-z
$$

The map (10) in our case is thus

$$
\left(\begin{array}{l}
\mathbf{u} \\
\mathbf{v}
\end{array}\right)=\left(\begin{array}{l}
P \\
y \\
z
\end{array}\right), \quad P=x(1-z g(x)) .
$$


If $r(\mathbf{x}, \mathbf{y})=x,(13)$ now yields

$$
x(y, z)=\sum_{\substack{k>0 \\(m, n) \in Z^{2}}} \operatorname{Res}\left\{x P^{-k-1} P^{\prime} y^{-m-1} z^{-n-1}\right\}(y+z)^{k} y^{m} z^{n},
$$

and it only remains to evaluate the residues.

Since $P$ does not depend on $y$, we need $m=0$ to obtain a residue in $y$. Using

$$
P^{-k-1} P^{\prime}=-\frac{1}{k}\left(P^{-k}\right)^{\prime},
$$

we thus get

$$
x(y, z)=-\sum_{\substack{k>0 \\ n \geqq 0}} \frac{1}{k} \operatorname{Res}_{x, z}\left\{x\left(P^{-k}\right)^{\prime} z^{-n-1}\right\}(y+z)^{k} z^{n},
$$

where the residue now is taken only with respect to the variables $x$ and $z$. In view of

$$
P=x(1-z g(x)),
$$

we may use the binomial series to obtain

$$
\begin{aligned}
p^{-k} & =x^{-k}(1-z g(x))^{-k} \\
& =x^{-k} \sum_{l=0}^{\infty}(-1)^{\prime}\left(\begin{array}{c}
-k \\
l
\end{array}\right) z^{\prime} g^{\prime},
\end{aligned}
$$

where $\left(\begin{array}{c}-k \\ l\end{array}\right)$ is a binomial coefficient. Now for given $k>0$ and $n \geqq 0$,

$$
\begin{aligned}
\operatorname{Res}_{x, z}\left\{x\left(P^{-k}\right)^{\prime} z^{-n-1}\right\} & =\operatorname{Res}_{x} \text { of coefficient of } z^{n} \text { in } x\left(P^{-k}\right)^{\prime} \\
& =-\operatorname{Res}_{x} \text { of coefficient of } z^{n} \text { in } P^{-k} \\
& =- \text { coefficient of } x^{k-1} \text { in }(-1)^{n}\left(\begin{array}{c}
-k \\
n
\end{array}\right) g^{n} \\
& =(-1)^{n+1}\left(\begin{array}{c}
-k \\
n
\end{array}\right) g_{k-1}^{(n)},
\end{aligned}
$$

where $\operatorname{Res}_{x}$ denotes the residue with respect to the single variable $x$, and where the coefficients $g_{k}^{(n)}$ are defined by

$$
[g(x)]^{n}=\sum_{k=0}^{\infty} g_{k}^{(n)} x^{k}
$$

We thus finally let

$$
\begin{aligned}
x(y, z) & =\sum_{\substack{k>0 \\
n \geqq 0}}^{\infty} \frac{(-1)^{n}}{k}\left(\begin{array}{c}
-k \\
n
\end{array}\right) g_{k-1}^{(n)}(y+z)^{k} z^{n} \\
& =y+z+\sum_{\substack{k>0 \\
n>0}} \frac{(-1)^{n}}{k}\left(\begin{array}{c}
-k \\
n
\end{array}\right) g_{k-1}^{(n)}(y+z)^{k} z^{n} .
\end{aligned}
$$


5. Truncation error. In numerical computation, the series (15) will have to be truncated, for instance, by neglecting the terms where $k+n \geqq p$ for some positive integer $p$. We therefore estimate the truncation error

$$
t_{p}(y, z)=\sum_{\substack{k+n=p \\
k>0, n>0}} \frac{(-1)^{n}}{k}\left(\begin{array}{c}
-k \\
n
\end{array}\right) g_{k-1}^{(n)}(y+z)^{k} z^{n} .
$$

From (6), the coefficients $a_{n}$ in

$$
[h(x)]^{-1}=\sum_{n=0}^{\infty} a_{n} x^{n}
$$

are easily seen to satisfy $\left|a_{n}\right| \leqslant 1$. In view of $a_{0}=1$ we therefore have for $|x| \leqslant \rho, \rho<1$,

$$
\left|[h(x)]^{-1}\right| \geqq 1-\rho-\rho^{2}-\cdots=(1-2 \rho) /(1-\rho),
$$

and thus, if $0 \leqq \rho<\frac{1}{2}$,

$$
|h(x)| \leqq(1-\rho) /(1-2 \rho) .
$$

Using the principle of the maximum, there follows for $|x| \leqq \rho<\frac{1}{2}$,

$$
|g(x)| \leqslant\left|\frac{1}{\rho}\left(\frac{1-\rho}{1-2 \rho}-1\right)\right|=\frac{1}{1-2 \rho} .
$$

Cauchy's estimate now yields

$$
\left|g_{k}^{(n)}\right| \leqq \frac{1}{(1-2 \rho)^{n}} \frac{1}{\rho^{k}}, \quad 0<\rho<\frac{1}{2} .
$$

Now let $|y+z| \leqq \rho_{1},|z| \leqq \rho_{2}$. In view of

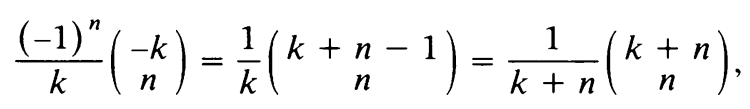

there follows

$$
\begin{aligned}
\left|\sum_{\substack{k+n=q \\
k>0, n>0}} \frac{(-1)^{n}}{k}\left(\begin{array}{c}
-k \\
n
\end{array}\right) g_{k-1}^{(n)}(y+z)^{k} z^{n}\right| & \leqq \frac{1}{q} \sum_{k+n=q}\left(\begin{array}{c}
q \\
n
\end{array}\right)(1-2 \rho)^{-n} \rho^{-k+1} \rho_{1}^{k} \rho_{2}^{n} \\
& =\frac{\rho}{q}\left(\frac{\rho_{2}}{1-2 \rho}+\frac{\rho_{1}}{\rho}\right)^{q} .
\end{aligned}
$$

Therefore, if

$$
\sigma=\frac{\rho_{2}}{1-2 \rho}+\frac{\rho_{1}}{\rho}<1,
$$

we find the truncation error estimate

$$
\left|t_{p}(y, z)\right| \leqq \frac{\rho}{p} \frac{\sigma^{p}}{1-\sigma} .
$$


Choosing, for instance, $\rho=\frac{1}{3}$, there results the simple formula

$$
\left|t_{p}(y, z)\right| \leqq \frac{1}{3 p} \frac{\left(3 \rho_{1}+3 \rho_{2}\right)^{p}}{1-\left(3 \rho_{1}+3 \rho_{2}\right)} .
$$

6. Numerical values. It remains to compute the coefficients $g_{k}^{(n)}$. This is a routine computation which is best performed with a symbolic manipulator. Using the MAPLE program of the University of Waterloo [2] we computed the $g_{k}^{(n)}$ as well as the coefficients

$$
a_{k}^{(n)}=\frac{(-1)^{n}}{k}\left(\begin{array}{c}
-k \\
n
\end{array}\right) g_{k}^{(n)}
$$

of the series (15) in rational arithmetic for $1 \leqq k \leqq 10,1 \leqq n \leqq 10$. Complete tables of these values are available from the authors on request. Here we give only the values that are required to write the terms of the series for $k+n<5$ :

$$
\begin{aligned}
h(x) & =1-\frac{9}{14} x-\frac{13}{392} x^{2}-\frac{4189}{181104} x^{3}-\cdots, \\
g(x) & =-\frac{9}{14}-\frac{13}{392} x-\frac{4189}{181104} x^{2}-\cdots, \\
{[g(x)]^{2} } & =\frac{81}{196}+\frac{117}{2744} x+\cdots, \\
{[g(x)]^{3} } & =-\frac{729}{2744}-\cdots .
\end{aligned}
$$

This results in

$$
\begin{aligned}
x(y, z)= & (y+z)\left\{1-\frac{9}{14} z+\frac{81}{196} z^{2}-\frac{729}{2744} z^{3}+\cdots\right\} \\
& +(y+z)^{2}\left\{-\frac{13}{392} z+\frac{351}{5488} z^{2}+\cdots\right\} \\
& +(y+z)^{3}\left\{-\frac{4189}{181104} z+\cdots\right\} \\
& +\cdots .
\end{aligned}
$$

From the values of the parameters given in [1] we have

$$
y=3.247890 \times 10^{-3}, \quad z=3.461391 \times 10^{-3} .
$$

Substituting these into (19) we get

$$
x=6.694379 \times 10^{-3}
$$

with a truncation error $t_{5}(y, z)$, which by (18) is less than

$$
\frac{1}{15} \frac{\left[3 \times 6.709281 \times 10^{-3}\right]^{5}}{0.979872}=2.25 \times 10^{-10},
$$

and which thus is less than the error in $x$ due to rounding or measuring errors in $y$ and $z$. 


\section{REFERENCES}

[1] J. C. Alexander, The numerics of computing geodetic ellipsoids, SIAM Rev. 27, 241-247 (1985)

[2] B. W. Char, K. O. Geddes, G. H. Gonnet, and S. M. Watt, Maple user's manual, 3rd ed., Univ. of Waterloo Res. Rep. CS-83-41 (1983)

[3] P. Henrici, Die Lagrange-Bürmannsche Formel bei formalen Potenzreihen, Jber. Deutsch. Math.-Verein. 86, $115-134(1984)$

[4] W. A. Hieskanen and H. Moritz, Physical geodesy, Freeman, San Francisco, 1966 